\title{
Incorporating the local biological effect of dose per fraction in IMRT inverse optimization
}

\author{
Brígida da Costa Ferreira ${ }^{1,2,5[0000-0001-7988-7545]}$, Panayiotis Mavroidis ${ }^{3,4}$, Joana Dias ${ }^{5[111-}$ \\ 2222-3333-4444] and Humberto Rocha ${ }^{5[1111-2222-3333-4444]}$ \\ ${ }^{1}$ School of Health, Polytechnic of Porto, Porto, Portugal \\ ${ }^{2}$ I3N Department of Physics, Aveiro University, Aveiro, Portugal \\ ${ }^{3}$ Department of Radiation Oncology, University of North Carolina at Chapel Hill, NC, USA \\ ${ }^{4}$ Division of Medical Radiation Physics, Karolinska Institutet and Stockholm University, \\ Stockholm, Sweden \\ ${ }^{5}$ Institute for Systems Engineering and Computers at Coimbra, Coimbra University, Coimbra, \\ Portugal \\ bcfeess.ipp.pt
}

\begin{abstract}
In intensity modulated radiation therapy (IMRT), the dose in each voxel of the organs at risk (OAR) can be strongly reduced compared to conformal radiation therapy (RT). Due to the sensitivity of late side-effects to fraction size, a smaller dose per fraction in the normal tissues represent an increased tolerance to RT. This expected reduction in biological effect may then be used as an additional degree of freedom during IMRT optimization. In this study, the comparison between plans optimized with and without a voxel-based fractionation correction was made. Four patients diagnosed with a head and neck (HN), a breast, a lung or a prostate tumor were used as test cases. Voxel-based fractionation corrections were incorporated into the optimization algorithm by converting the dose in each normal tissue voxel to EQD2 (equivalent dose delivered at 2 Gy per fraction). The maximum gain in the probability of tumor control $\left(P_{\mathrm{B}}\right)$, due to the incorporation of the correction for fractionation in each voxel, was $1.3 \%$ with a $0.1 \%$ increase in the probability of complications $\left(P_{\mathrm{I}}\right)$ for the HN tumor case. However, in plan optimization and evaluation, when tolerance doses were compared with the respective planned EDQ2 (calculated from the 3-dimensional dose distribution), $P_{\mathrm{B}}$ increased by $19.3 \%$ in the $\mathrm{HN}, 12.5 \%$ in the lung, $6.2 \%$ in the breast and $2.7 \%$ in the prostate tumor case, respectively. The corresponding increases in $P_{\mathrm{I}}$ were $2.3 \%, 6.2 \%, 1.0 \%$ and $0.7 \%$, respectively. Incorporating voxelbased fractionation corrections in plan optimization is important to be able to show the clinical quality of a given plan against established tolerance constraints. To properly compare different plans, their dose distributions should be converted to a common fractionation scheme (e.g. 2 Gy per fraction) for which the doses have been associated with clinical outcomes.
\end{abstract}

Keywords: Radiation Therapy, IMRT optimization, voxel-based fractionation corrections. 


\section{Introduction}

Historically, the dose tolerances to RT of the OAR were mostly derived from dose distributions irradiating tissues homogeneously with the conventional fractionation of 2 Gy per fraction. Tabulated dose tolerance values are closely followed during treatment plan optimization as if the organ would be irradiated homogeneously or with the same dose distribution as those patient who were used to derive those tolerance values. However, with 3D Conformal RT and more recently with IMRT, most OAR are heterogeneously irradiated with large portions of their volumes receiving fractional doses lower than 2 Gy. Additionally, the tri-dimensional dose distributions vary greatly between patients and alternative fractionations are becoming increasingly used, (e.g. the use of simultaneous integrated boost techniques have become the method of choice to deliver IMRT) [1]. Due to this variety of fractionation schedules and scarcity of patient clinical data, tolerance doses are derived from converting all delivered treatments to a 2 Gy fractionation schedule.

Late RT side-effects are radiosensitive to fraction size. Thus, normal tissue voxels irradiated with a dose per fraction much lower than $2 \mathrm{~Gy}$ will have a larger tolerance to radiation than those regions irradiated with doses equal or higher than $2 \mathrm{~Gy}$. With IMRT, high dose gradients are produced in the target volume borders and OAR located outside the planning target volume (PTV) will be irradiated with maximum fractional doses much smaller than 2 Gy. For instance, in a HN cancer patient prescribed to 70.2 Gy in 33 fractions, the tolerance dose of 45 Gy for the spinal cord was previously used [1]. In this case, the maximum dose per fraction in that organ will be $1.36 \mathrm{~Gy}$ and the tolerance dose would be $49.5 \mathrm{~Gy}(\alpha / \beta$ of $3 \mathrm{~Gy}$ ). For parallel organs the correction of the tolerance dose for fractionation is not as straightforward, as the biological effect depends on the 3D dose distribution irradiating the organ. During plan optimization and evaluation, the comparison between tabulated tolerance dose values and the planned dose for each structure should therefore be done using a common fractionation scheme.

In particularly difficult cases needing to improve the probability of tumour cure, taking into account the dose per fraction at the voxel level may be an additional degree of freedom during inverse IMRT optimization to improve the quality of RT. Increased normal tissue tolerance to radiation due to the heterogeneity in the fractional dose map, associated with a reduced dose in the OAR obtained with typical IMRT dose distributions, may allow the improvement in target volume coverage and thus treatment outcome.

In this study, a comparison between treatment plans, which were optimized with and without voxel-based fractionation correction was made. In this context, the physical dose loses its meaning and plan evaluation should then be based on EQD2 values. The gain obtained with this approach was then also quantified.

\section{$2 \quad$ Material and methods}

Four test patients diagnosed with HN, breast, lung or prostate tumours were included in this study. All cases were planned with simultaneous integrated boost techniques 
using 7 or 9 equidistant beams, except for the breast tumour case where 5 beams placed around the target breast, were used. In the HN tumour case, the prescription dose was 70.2 Gy and 63.0 Gy delivered in 33 fractions to the gross disease (primary tumour and adenopathies, PTV-T) and high risk lymph nodes (PTV-N), respectively. In the lung tumour case, the prescription dose was $70 \mathrm{~Gy}$ and $56 \mathrm{~Gy}$ to gross disease and enlarged planning target volume, respectively delivered in 31 fractions. In the breast case, the prescription dose was 66 Gy to the tumour bed and $50 \mathrm{~Gy}$ to the all breast delivered in 25 fractions; and in the prostate tumour case, the dose prescription was $74.2 \mathrm{~Gy}$ and 56.0 Gy to gross disease and involved lymph nodes, respectively. The tolerance doses followed Emami et al 2013 [2] recommendations, except for the spinal cord where the tolerance dose of 45 Gy was used [1].

Table 1. Dose-response parameters for the tumor cases used in this study.

\begin{tabular}{|c|c|c|c|c|c|}
\hline ROI & $D_{50} / G y$ & $\gamma$ & ROI & $D_{50} / G y$ & $\gamma$ \\
\hline & \multicolumn{2}{|c|}{ Head and neck } & \multicolumn{3}{|c|}{ Prostate } \\
\hline GTV-T & 55.0 & 8.0 & Prostate & 74.0 & 7.5 \\
\hline PTV-T & 54.0 & 7.5 & PTV-T & 73.0 & 6.0 \\
\hline PTV-N & 46.0 & 4.8 & PTV-N & 42.0 & 3.5 \\
\hline \multicolumn{3}{|c|}{ Lung } & \multicolumn{3}{|c|}{ Breast } \\
\hline GTV & 52.0 & 7.5 & Tumor bed & 50.0 & 4.0 \\
\hline CTV & 50.0 & 5.0 & CTV & 35.0 & 2.0 \\
\hline PTV & 40.0 & 4.0 & PTV & 30.0 & 1.0 \\
\hline
\end{tabular}

* GTV is gross tumor volume and CTV is the clinical target volume

Corrections for fractionation were performed in each voxel of the normal tissues, for each plan obtained in each optimization iteration, using the Biologically Effective Dose (BED) concept described in detail elsewhere [1]. $\alpha / \beta$ values of 10.0 were used for the $\mathrm{HN}$ and the lung tumours, 4.0 for the breast tumour and 3.0 for the prostate tumour and OARs. Repopulation was considered using a potential doubling time of 3 days and kickoff time of 28 days for the HN cancer, 5 days and 14 days for the lung cancer, and 4 days and 29 days for the prostate tumour, respectively. For the breast tumour case a potential doubling time of 15 days was used $[3,4,5,6]$.

Plan comparison was made using conventional dose statistics and radiobiological metrics such as the uncomplicated tumour control probability $\left(P_{+}\right)$, the probability of tumour control $\left(P_{\mathrm{B}}\right)$ and the probability of normal tissue complications $\left(P_{\mathrm{I}}\right)$. The relative seriality model and the linear-quadratic-Poisson model were used to determine the probability of response of the OARs and targets [7]. The model parameters used for each tumour volume are listed in Table 1. The dose-response parameters for the OARs are summarized in Mavroidis et al [8].

The open access treatment planning system matRad (developed by DKFZ) was used for inverse IMRT optimization [9]. The voxel-based fractionation correction was additionally implemented into the code by identifying the target or OAR to which each 
voxel belongs. Thus, the evaluation of the objective function was based on the dose matrix converted to $2 \mathrm{~Gy}$, instead of the physical dose matrix, to drive the optimization algorithm. Furthermore, the calculation of the probabilities of tumour cure and normal tissue complication were incorporated in this system.

Three plans were simulated for each patient: 1) the initial plan, which was conventionally optimized based on physical dose (noVC); 2) a new plan which was re-optimized using the voxel-based fractionation correction and the same objective function, i.e., no adjustments were made on objectives and penalties (VC); and 3) a final plan, using the voxel-based fractionation correction, which was re-optimized adjusting objectives and penalties based on planned biological dose (VC+EQD2).

\section{Results}

By comparing $\mathrm{VC}$ with noVC, the maximum gain in the probability of tumor control for the patient with an $\mathrm{HN}$ tumor, was $1.3 \%$ with a $0.1 \%$ increase in the probability of complications (Table 2). This was mostly due to an improvement in the irradiation of PTV-N. No significant differences in the dosimetry or in the response probabilities of the OARs were obtained except for a small increase in the minimum and mean dose in the oral cavity, which however did not result in an increase in the probability of injury.

When plan optimization and evaluation was based on planned EDQ2 values (VC+EQD2), the probability of tumor control increased by $19.3 \%$ in the HN tumor. This was mostly due an 9.5 Gy increase in the near-minimum biological dose in PTV$\mathrm{N}$ and about $3 \mathrm{~Gy}$ in PTV-T. This resulted in an increase of less than $0.6 \%$ in the probability of xerostomia and mucositis.

The most critical organ at risk in HN cancer RT is the spinal cord. For the plan noVC, the spinal cord had a maximum physical dose of $44.5 \mathrm{~Gy}$ and an EQD2 value of 38.7 Gy given thus some freedom to improve tumor coverage. Thus, in plan VC+EQD2 the probability of tumor control increased from $61.7 \%$ to $81.0 \%$ for a final EQD2 value to spinal cord of $44.5 \mathrm{~Gy}$ (physical dose of $49.5 \mathrm{~Gy}$ ).

When applying the voxel-based fractionation correction by itself negligible benefits were obtained for the other tumor cases. For the lung, breast and prostate tumor case, the difference in $P_{\mathrm{B}}$ between the plan VC and noVC were $0.3 \%, 0.02 \%$ and $-0.3 \%$. However, when the optimization was based on EQD2 planned values, gains in $P_{+}$of $6.4 \%, 5.2 \%$ and $1.9 \%$ for the lung, breast and prostate tumor, respectively, were obtained due to increased $P_{\mathrm{B}}$ by $12.5 \%, 6.2 \%$ and $2.7 \%$ and in $P_{\mathrm{I}}$ by $6.2 \%, 1.0 \%$ and $0.7 \%$, respectively.

Table 2. Results for the HN cancer case. The first group of columns report the difference between the results obtained with plan VC and plan noVC. The second group of columns report the differences between plan VC+EQD2 and noVC. Dosimetry reports differences in biological the dose, i.e., corrected to a dose per fraction of $2 \mathrm{~Gy}$.

\begin{tabular}{l|ccc|ccc}
\hline ROI & \multicolumn{3}{c}{$\mathrm{VC}-$ noVC } & \multicolumn{3}{c}{ VC+EQD2 - noVC } \\
\hline & $\Delta P_{+} / \%$ & $\Delta P_{\mathrm{B}} / \%$ & $\Delta P_{\mathrm{I}} / \%$ & $\Delta P_{+} / \%$ & $\Delta P_{\mathrm{B}} / \%$ & $\Delta P_{\mathrm{I}} / \%$ \\
\hline
\end{tabular}




\begin{tabular}{l|cccc|cccc}
\hline & 1.1 & 1.3 & 0.1 & & 17.0 & 19.3 & 2.3 & \\
\hline & $\boldsymbol{\Delta} \boldsymbol{P}_{\mathbf{B} / \mathbf{I} / \boldsymbol{\%}}$ & $\boldsymbol{\Delta} \boldsymbol{D} \mathbf{9 8} / \mathbf{G} \mathbf{y}$ & $\boldsymbol{\Delta} \boldsymbol{D}_{\text {mean }} / \mathbf{G y}$ & $\boldsymbol{\Delta} \boldsymbol{D}_{\mathbf{2}} / \mathbf{G} \mathbf{y}$ & $\boldsymbol{\Delta} \boldsymbol{P}_{\mathbf{B} / \mathbf{I} / \boldsymbol{\%}}$ & $\boldsymbol{\Delta} \boldsymbol{D} \mathbf{9 8} / \mathbf{G y}$ & $\boldsymbol{\Delta} \boldsymbol{D}_{\text {mean }} / \mathbf{G y}$ & $\boldsymbol{\Delta} \boldsymbol{D}_{\mathbf{2}} / \mathbf{G} \mathbf{y}$ \\
\hline GTV-T & 0.1 & 0.2 & 0.1 & -1.7 & 1.4 & 3.1 & 1.6 & 1.9 \\
PTV-T & 0.0 & 0.2 & 0.0 & -0.1 & 1.1 & 3.8 & 1.5 & 1.8 \\
PTV-N & 1.3 & 1.1 & 0.2 & -0.1 & 18.6 & 9.5 & 3.6 & 3.0 \\
Spinal cord & 0.0 & 0.0 & 0.4 & -0.3 & 0.0 & 0.0 & 4.0 & 5.6 \\
Ips. Parotid & 0.0 & 0.0 & -0.1 & 0.4 & 0.6 & 0.3 & 5.3 & 9.4 \\
Ctr.Parotid & 0.0 & -0.4 & 0.0 & 1.5 & 0.3 & 0.6 & 4.3 & 7.7 \\
Oral cavity & 0.0 & 2.4 & 3.0 & 1.0 & 0.1 & 0.4 & 3.5 & 3.1 \\
RVR & 0.1 & 0.0 & 0.2 & 0.0 & 1.2 & 0.0 & 0.5 & 1.9 \\
\hline
\end{tabular}

* $D_{98}$ and $D_{2}$ is the near-minimum and near-maximum dose, respectively and $D_{\text {mean }}$ is the mean dose.

The improvements obtained in the lung cancer case with the plan VC+EQD2, compared to noVC plan, were mostly due to improvements in the probability of tumor control of the external PTV and CTV by $9.5 \%$ and $5.0 \%$, respectively, due to an increase of about $6 \mathrm{~Gy}$ in the near-minimum dose in these volumes. Simultaneously, an increase in the probability of injury in the ipsilateral lung by $5 \%$ was obtained. The difference in the probability of RT side-effects for the heart, liver, spinal cord and esophagus between VC+EQD2 and noVC was below $1 \%$.

Similarly, for the breast cancer case, the largest improvements were obtained by the better CTV and PTV irradiation increasing the probability of tumor control from $58.9 \%$ with noVC to $65.1 \%$ with VC+EQD2 while the increase in probability of complications was very small (1\%). The probability of complications in heart, lungs, and contralateral breast remained almost the same.

For the prostate cancer case, the gains by using these two new approaches were small. The gain in using EQD2 values for plan evaluation, compared to conventional optimization, was of about $1.8 \%$ for both PTVs due to an increase in the minimum dose in these volumes while the difference in the probability of complications in the rectum, bladder and femoral heads between the two methods remained below $0.4 \%$.

\section{Discussion}

The heterogeneity obtained with IMRT dose distributions, which reduces not only the total dose but also the dose per fraction delivered to the OARs, suggests that there might be room for improving target volume coverage. The numerical correction of tabulated tolerance doses to different fractionation schedules is not sufficient during IMRT planning as the dose distribution changes in each optimization iteration. Furthermore, incorporating voxel-based fractionation corrections allows a compatible comparison between planned doses and established tolerances doses using a common fractionation scheme. 
In this study, when this correction was incorporated into the optimization algorithm, the gain, obtained by just re-optimizing the plan without adjusting any of the objectives and penalties, was small. This comparison demonstrates the shortcomings of evaluating plan quality based on physical dose objectives and constraints. This emulates clinical procedures that do not take into account the full potential of RT fractionation. This can be though as a reverse double-trouble effect that is being overlooked.

When plan optimization and evaluation compared plans, which were produced based on fractionation corrected biological dose against plans produced based on physical tolerance doses, the gain in probability of uncomplicated tumour control was substantial especially for the most difficult tumours cases. The reduction in dose per fraction in the normal tissues allowed to mostly escalate the minimum dose in the (outer) target volume significantly improving the probability of tumour control. Inevitably this also resulted in an increase in the probability of complications. However, the gain obtained in the probability of tumour control outweighed the loss in the probability of RT sideeffects.

For tumour cases with OARs partially located in the PTV, irradiated with simultaneous integrated boost techniques, the effect is the opposite as described before as these OARs will be irradiated with a dose per fraction larger than $2 \mathrm{~Gy}$. In this case, voxelbased optimization algorithms will take this into account investing its efforts in trying to reduce the dose in those voxels.

This work followed the assumption that each OAR is characterized by a homogenous radiosensitivity. However, the ability to determine the internal organization of the OARs in a more precise and quantitative manner would bring additional benefits for treatment planning. Normal tissue response to RT needs to be thoroughly investigated to maximize treatment individualization and the success of delivered therapies.

\section{References}

1. Ferreira, B.C.; Lopes, M.C.; Mateus, J.; et al: Radiobiological evaluation of forward and inverse IMRT using different fractionations for head and neck tumours. Radiat. Oncol 5:57 (2010).

2. Emami, B.; Lyman, J.; Brown, A.; et al: Tolerance of normal tissue to therapeutic irradiation. Int. J. Radiat. Oncol. Biol., Phys. 21, 109-122 (1991).

3. Wyatt, R.M.; Beddoe A.H.; Dale R.G.. The effects of delays in radiotherapy treatment on tumour control. Phys Med Biol. 48(2):139-55 (2003).

4. Partridge, M.; Ramos, M.; Sardaro, A.; Brada, M.. Dose escalation for non-small cell lung cancer: analysis and modelling of published literature. Radiother Oncol. 99(1):6-11 (2011).

5. Pedicini, P.; Strigari, L.; Benassi M. Estimation of a self-consistent set of radiobiological parameters from hypofractionated versus standard radiation therapy of prostate cancer. Int $\mathbf{J}$ Radiat Oncol Biol Phys. 1;85(5):e231-7 (2013).

6. Fowler JF: Optimum overall times II: Extended Modelling for head and neck radiotherapy. Clinical Oncol 20:113-126 (2008)

7. Källman, P.; Ågren, A.; Brahme, A. 1992 Tumour and normal tissue responses to fractionated non-uniform dose delivery. Int J Radiat Biol. 62(2):249-62. 
8. Mavroidis, P.; Ferreira, B.C.; Papanikolaou, N.; Lopes, M.C. Analysis of fractionation correction methodologies for multiple phase treatment plans in radiation therapy. Med Phys. 40(3):031715 (2013).

9. Wieser, H.P.; Cisternas, E.; Wahl, N. et al: Development of the open-source dose calculation and optimization toolkit matRad. Med Phys. 44(6):2556-2568 (2017). 